\title{
A Suggestion to Make Thermodynamic Theory More Easily Understandable
}

\author{
Jean-Louis Tane \\ Formerly with the Department of Geology, University of Grenoble, Grenoble, France \\ Email: tanejl@aol.com
}

How to cite this paper: Tane, J.-L. (2021) A Suggestion to Make Thermodynamic Theory More Easily Understandable. Journal of Applied Mathematics and Physics, 9, 920-930. https://doi.org/10.4236/jamp.2021.95062

Received: April 12, 2021

Accepted: May 16, 2021

Published: May 19, 2021

Copyright $\odot 2021$ by author(s) and Scientific Research Publishing Inc. This work is licensed under the Creative Commons Attribution International License (CC BY 4.0).

http://creativecommons.org/licenses/by/4.0/

\begin{abstract}
The conceptual difficulties encountered in thermodynamics are well known and are certainly the reasons that have led the great physicist Arnold Sommerfeld, a long time ago, to say that understanding thermodynamics is not easy. The situation remains nearly the same today and is due to the fact that the tools used in thermodynamics, i.e. the equations, are not in good accordance with the laws of thermodynamics. Since the efficiency of the tools cannot be contested, it is probably the formulation of the laws that needs to be revised. On the basis of arguments already evoked in previous papers, the suggestion presented below is a contribution going in this sense and inserting the Einstein's relation $E=m c^{2}$ in the thermodynamic reasoning.
\end{abstract}

\section{Keywords}

Thermodynamics, Reversibility, Irreversibility, Energy, Entropy, Einstein's Mass-Energy Relation, Relativity, Gravitation

\section{Introduction}

The scientific reputation of Arnold Sommerfeld seems to rest in two main reasons. The first one is that he has been the scientist the most often nominated for the Nobel Prize in Physics (81 times according to Wikipedia) although he never won it. The second is that his judgment on thermodynamics remained famous because of the next shape he gave it: "Thermodynamics is a funny subject. The first time you go through it, you don't understand it at all. The second time you go through it, you think you understand it, except for one or two points. The third time you go through it, you know you don't understand it, but by that time you are so used to the subject, it doesn't bother you anymore". Confronting both reasons, it cannot be excluded that some members of the Nobel Prize Committee have not appreciated the judgment given by Arnold Sommerfeld about thermo- 
dynamics. What seems sure is that his qualities as professor and research director were appreciated by his students and have certainly contributed to the fact that several of them received the Nobel Prize in physics. This was the case for Werner Heisenberg (1932), Peter Debye (1936) and Wolfgang Pauli (1945).

As pointed out by D. K. Nordstrom and J. L. Munoz in the preface of their own book dealing with thermodynamics (number 2 of the section References), the expression "it doesn't bother you anymore" is a way to say that the thermodynamic tool can be used successfully even if the corresponding theory is felt unclear. Despite the reassuring aspect of this information, the aim of the present paper is focused on the need to clarify the theory.

\section{A Brief Reminder on the First Law of Thermodynamics}

Let us imagine that the system we are considering (Figure 1) is a given mass of gas placed in a glass cylinder, equipped with a mobile frictionless piston. In the initial state, the piston is in equilibrium at height $\mathrm{A}$ and the first equation we write takes the form:

$$
P_{i 1}=P_{e 1}
$$

whose meaning is:

Internal pressure at time $1=$ External pressure at time 1 .

More precisely, this is a way to say that the pressure exerted on the gas (i.e. the atmospheric pressure augmented by the pressure due to the mass of the piston) is equal to the pressure exerted by the gas on the inderside of the piston.

If a determined mass $M$ of sand is deposited on the piston, this last one goes downward until it reaches a new equilibrium state at height $\mathrm{B}$, where the new equation of equilibrium becomes:

$$
P_{i 2}=P_{e 2}
$$

Depending on whether the mass of sand is deposited all at once or in successive small batches, the time required by the piston to go down from $A$ to $B$ is not the same.

In the first case, the external pressure $P_{e}$ and the internal pressure $P_{i}$ are constantly different during the moving of the piston and become equal only when the piston stops. This condition is called irreversibilty with regard to the pressure and the work done on the gas by the piston is defined at each moment by
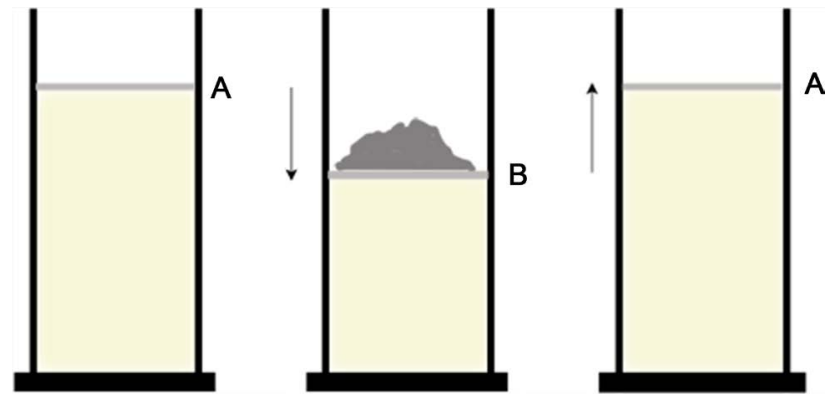

Figure 1. Schematic view of the experimental context. 
the relation:

$$
d W_{i r r}=-P_{e} d V
$$

where $d V$ is the volume change of the gas.

In the second case, the external pressure $P_{e}$ and the internal pressure $P_{i}$ tend to be constantly equal during the moving of the piston. This condition is called reversibilty with regard to the pressure and the work done on the gas by the piston is defined at each moment by the relation:

$$
d W_{\text {rev }}=-P_{i} d V
$$

For a given value of $d V$, the difference between $d W_{i r r}$ and $d W_{\text {rev }}$ is therefore:

$$
d W_{\text {irr }}-d W_{\text {rev }}=d V\left(P_{i}-P_{e}\right)
$$

that can also be written:

$$
d W_{i r r}=d W_{r e v}+d V\left(P_{i}-P_{e}\right)
$$

The condition $\left(P_{i}-P_{e}\right)>0$ leading to $d V>0$ and the condition $\left(P_{i}-P_{e}\right)<0$ to $d V<0$, we have always $d V\left(P_{i}-P_{e}\right)>0$, and therefore the relation between $d W_{i r r}$ and $d W_{r e V}$ is always:

$$
d W_{i r r}>d W_{\text {rev }}
$$

Having in mind the representation given above (Figure 1), we easily conceive that when $P_{i}$ and $P_{e}$ are rigorously equal, the piston does not move, so that we get $d V=0$. The evidence of this situation is well known and has inspired to Arnold Sommerfeld the following comment: Reversible processes are not, in fact, processes at all, they are sequences of states of equilibrium. The processes which we encounter in real life are always irreversible processes. This is a way to recall that the condition of reversibility is a limited case, whose usefulness is mainly theoretical. From the practical point of view, the information to keep in mind is that when a system passes from a state $\mathrm{A}$ to a state $\mathrm{B}$, depending on whether the process is highly irreversible or slightly irreversible (with regard to the pressure), the physical significance of Equation (7) is:

$$
d W_{\text {irr highly }}>d W_{\text {irr slightly }}
$$

In conditions of irreversibility, it has been observed for a long time that when the piston moves down (case represented by the left arrow), the temperature of the gas tends to increase temporarily, before returning to its initial value. This is the sign that a part of the work done on the gas is transformed in heat, classically noted $d Q$. But this heat is finally eliminated towards the surroundings so that its writing takes the negative form:

$$
d Q<0
$$

Conversely, if the sand initially deposited is removed, the piston will moves up (case represented by the right arrow) and it has been observed that the temperature of the gas decreases temporarily, before returning to its initial value. This is the sign that a given amount of heat has transited from the surroundings (the atmosphere) to the gas. Being received by the system, this heat is counted posi- 
tively through the writing:

$$
d Q>0
$$

In the process just examined, the energy given or removed by the experimentor is exclusively under the form of work (by deposit or evacuation of sand). As a consequence, the appearance or disappearance of heat is a natural reaction entirely due to the system itself. In many other contexts, the experimentor plays directly a role on both work and heat (or only on heat as will be the case in section 3 and 4).

The numerous studies done on this subject have led to the first law of thermodynamics. With reference to the simple experimental context considered here, it consists in admitting as a general postulate that the concept of internal energy variation called $d U$ and defined by the relation:

$$
d U=d Q+d W
$$

is independent of the level of irreversibility of the process by which the system passes from an initial state A to a final state B. (The fact that, in Equation (11), $d Q$ is placed before $d W$ and not after is just due to the respect of a thermodynamic writing convention).

In other words, this is a way to say that the term $d U$ always obeys the relation:

$$
d U_{i r r}=d U_{r e v}
$$

Having got for $d W$ the general proposal $d W_{i r r}>d W_{\text {rev }}$ given by Equation (7), the logic of the thermodynamic reasoning would be that the proposal obtained for $d Q$ should take the form:

$$
d Q_{i r r}<d Q_{\text {rev }}
$$

The next section is devoted to this question.

\section{A Brief Reminder of the Second Law of Thermodynamics}

In the same manner as Equations (3) and (4) show the link existing between the terms $d W, P$ and $d V$, a thermodynamic link has been established between $d Q, T$ and the term $d S$, called the change in entropy.

This last concept being less familiar than that of change in volume $(d V)$ it is important to begin the discussion with a reminder of the definition given to $d S$ in thermodynamics.

In condition of reversibility (i.e. when we have constantly $T_{i}=T_{e}$ ), the term $d S$ is linked to $d Q$ and $T$ (the absolute temperature) by the relation:

$$
d S=d Q / T
$$

In conditions of irreversibility (i.e. when we have $T_{i} \neq T_{e}$ ), the term $d S$ is linked to $d Q$ and $T$ (the absolute temperature) by the relation:

$$
d S>d Q / T
$$

This last relation is often written:

$$
d S=d Q / T+d S_{i}
$$


where $d S_{i}$ is called the internal component of entropy and has always a positive value.

In contrast, the term $d Q / T$ is called the external component of entropy and noted $d S_{e}$, so that Equation (16) takes the significance:

$$
d S=d S_{e}+d S_{i}
$$

This equation illustrates the fact that, in conditions of irreversibility, a part of the entropy, noted $d S_{e}$, is due to the exchange of heat between the system and its surroundings, while another part, noted $d S_{i p}$ is created inside the system.

Having in mind that the result $d Q_{i r r}<d Q_{r e v}$ given by Equation (13) was presented as the logical consequence of the first law of thermodynamics, we may be tempted to think that the term $d Q$ of Equation (14) represents $d Q_{r e r}$ while the term $d Q$ of relation 15 and Equation (16) represents $d Q_{i r r}$ In such conditions, the coherency of the theory would seem reached.

The problem is that this impression is not the adequate one and there are two reasons to this situation:

The first is that the precise meaning of Equation (14) is:

$$
d S=d Q_{r e v} / T_{i}
$$

The second is that the precise meaning of relation (15) is:

$$
d S>d Q_{r e v} / T_{e}
$$

Consequently, the precise meaning of Equation (16) becomes:

$$
d S=d Q_{r e v} / T_{e}+d S_{i}
$$

Combining Equations (18) and (20), we can see that for a given value of $d S$, the corresponding value of $d S_{i}$ is given by equation:

$$
d S_{i}=d Q_{\text {rev }}\left(\frac{1}{T_{i}}-\frac{1}{T_{e}}\right)
$$

The positive value of $d S_{i}$ (evoked in the comment following Equation (16)) is classically confirmed by Equation (21) through the fact its right end term is always positive.

Indeed, when we have $T_{i}<T_{e}$, the term in parentheses is positive and $d Q_{r e v}$ too.

When we have $T_{i}>T_{e}$, the term in parentheses is negative and $d Q_{r e v}$ too.

Therefore the general result that needs to be retained takes the form:

$$
d S_{i}>0
$$

It constitutes a simple expression of the second law of thermodynamics as well as Equation (11) ( $d U=d W+d Q)$ with the conditions already mentioned is a simple expression of the first law. Equation (22) expresses the fact that when a thermodynamic system evolves from an initial state A to a final state B, the internal component of its entropy (term $d S_{i}$ of Equation (17)) always goes increasing. 


\section{The Divergence between Theory and Practice in Thermodynamics}

At this point of the discussion, the attention is called on the fact that:

- we are reasoning on systems as simple as possible

- we have in mind the judgment beared by Arnold Sommerfeld on thermodynamics

- we are trying to test the coherency of the theory, which means that, according to the usual understanding of the first law, we want to see if the proposal $d Q_{i r r}<d Q_{r e v}$ suggested by Equation (13), is confirmed.

Observing that we can deduce from Equation (18) the relation:

$$
d Q_{\text {rev }}=T_{i} d S
$$

our remaining objective is the search of the corresponding relation concerning $d Q_{\text {irr }}$

In the list of 23 equations already written, the term $d Q_{i r r}$ is present nowhere, excepted in Equation (13) which is not an answer, but just a question.

Confronted to this situation, it seems that the only possible solution to get $d Q_{\text {irr }}$ consists in multiplying both sides of relation 20 , which is an entropy equation, by $T_{e}$, and we obtain the energy equation:

$$
T_{e} d S=d Q_{r e v}+T_{e} d S_{i}
$$

whose thermodynamic meaning is:

$$
d Q_{i r r}=d Q_{r e v}+T_{e} d S_{i}
$$

Knowing from Equation (21) that we have $d S_{i}>0$ and having also $T_{e}>0$ since $T_{e}$ is an absolute temperature, the obtained conclusion takes the form:

$$
d Q_{i r r}>d Q_{r e v}
$$

Obviously, this result is not compliant with the one usually predicted by the first law of thermodynamics and therefore requires an explanation. Before discussing this interesting question in section 5, let us examine a very simple example of calculus leading to a confirmation of Equation (26).

The system we are considering is 1 liter of water $(=1000 \mathrm{~g})$ placed in a transparent graduate cylinder. In the initial state $\mathrm{A}$, the temperature of the water is $25^{\circ} \mathrm{C}(=298 \mathrm{~K})$ and by heating the water passes to a final state $\mathrm{B}$ where its temperature is $80^{\circ} \mathrm{C}(=353 \mathrm{~K})$. Our objective is the determination of the various terms evoked above: $\Delta Q_{\text {irr }}, \Delta Q_{\text {rev }} \Delta S, \Delta S_{e}$ and $\Delta S_{i}$.

A first important remark is that inside the transparent cylinder, the water level did not change by heating. This seems the sign that the water volume does not change either so that we don't have to consider the existence of a term $\Delta W$.

As a consequence we can deduce from Equation (11) that the change in internal energy of the water is limited to the expression:

$$
\Delta U=\Delta Q
$$

Then, remembering that the first law postulates the equality:

$$
\Delta U_{\text {irr }}=\Delta U_{\text {rev }}
$$


the conclusion theoretically expected would take the form:

$$
\Delta Q_{i r r}=\Delta Q_{r e v}
$$

As will be seen below, the terms $\Delta Q_{i r r}$ and $\Delta Q_{r e v}$ are not equal and therefore are not in accordance with the usual interpretation of the first law.

For the calculation of $\Delta Q_{\text {rev }}$ the equation classically used is:

$$
\Delta Q_{\text {rev }}=\int_{298}^{353} m c_{p} d T
$$

Considering that $m$ and $c_{p}$ can be admitted constant over the temperature range, we get:

$$
\begin{gathered}
\Delta Q_{r e v}=m c_{p} \int_{298}^{353} d T=1000 \times 4.18 \times(353-298)=229900 J \\
\Delta S=m c_{p} \int_{298}^{353} \frac{d T}{T}=1000 \times 4.18 \times \ln \frac{353}{298}=708 \mathrm{~J} \cdot \mathrm{K}^{-1}
\end{gathered}
$$

If, in a second experiment, the cylinder containing the water at $298 \mathrm{~K}$ is itself immersed in a larger water tank whose temperature is $353 \mathrm{~K}$, this temperature becomes the term $T_{e}$ (the external temperature) evoked in Equation (24). This is a way to say that, according to the relation linking together Equations (24) and (25) $\left(\Delta Q_{i r r}=T \Delta S\right)$, the value of the term $\Delta Q_{i r r}$ is:

$$
\Delta Q_{i r r}=T_{e} \Delta S=353 \times 708=249224 \mathrm{~J}
$$

This last result corresponds effectively to the condition $d Q_{i r r}>d Q_{\text {rev }}$ and not to the condition $d Q_{i r r}=d Q_{r e v}$ evoked by Equation (29) and presented as the logical prediction of the first law.

In this conception, the difference between $\Delta Q_{i r r}$ and $\Delta Q_{\text {rev }}$ appears as an additional energy that can be called $\Delta Q_{a d d}$ and whose definition is:

$$
\Delta Q_{\text {add }}=\Delta Q_{\text {irr }}-\Delta Q_{\text {rev }}
$$

Its numerical value, in the present case, is:

$$
\Delta Q_{a d d}=249224-229900=19324 \mathrm{~J}
$$

The other expected results are as follows:

$$
\begin{aligned}
\Delta S_{e} & =\frac{\Delta Q_{\text {rev }}}{T_{e}}=\frac{229900}{353}=651 \mathrm{~J} \cdot \mathrm{K}^{-1} \\
\Delta S_{i} & =\Delta S-\Delta S_{e}=708-651=57 \mathrm{~J}
\end{aligned}
$$

\section{Discussion}

The main difference between the conventional interpretation of the first law of and the new suggested one can be summarized as follows:

In the conventional interpretation, the entropy Equation (20) whose expression is:

$$
d S=d Q_{\text {rev }} / T_{e}+d S_{i}
$$

is not converted into the energy Equation (24) whose expression is:

$$
T_{e} d S=d Q_{r e v}+T_{e} d S_{i}
$$


and whose meaning is:

$$
d Q_{i r r}=d Q_{r e v}+d Q_{a d d}
$$

From Equations (24) and (38), it can be seen that the definition of $d Q_{\text {irr }}$ takes the form:

$$
d Q_{\text {irr }}=T_{e} d S
$$

It constitutes the expected complement evoked in the line following Equation (23), which was itself devoted to the term $d Q_{\text {rer }}$ through the definition:

$$
d Q_{\text {rev }}=T_{i} d S
$$

Comparing both definitions, the conclusion $d Q_{i r r}>d Q_{\text {rev }}$ can be deduced by the same kind of simple reasoning as the conclusion $d W_{i r r}>d W_{\text {rev }}$ obtained above from Equation (7).

Transposed in the context of change in internal energy, the term $d Q_{a d d}$ of Equation (38) becomes a contribution to the term $d U_{\text {add }}$ of the energy equation:

$$
d U_{i r r}=d U_{r e v}+d U_{\text {add }}
$$

explaining that the formulation of the first law would imply the inequality:

$$
d U_{i r r}>d U_{r e v}
$$

instead of the equality $d U_{\text {irr }}=d U_{\text {rev }}$ usually admitted.

Concerning this question, the attention is called on the two following points.

- An irreversibility of pressure generates a heat that is really detected before being ejected from the system (context examined above in section 2);

- An irreversibility of temperature generates an energy which seems implicitly evident but is not really detected (context examined above in section 3 and 4).

It is certainly for this second reason that, when the thermodynamic theory was developed ( $19^{\text {th }}$ century), the term $d S_{i}$ of equation 20 has not been identified as a real symptom of energy creation, despite the fact that, from the mathematical point of view, the conversion of the entropy equation 20 into the energy equation 24 don't raises problem. The conceptual difficulties evoked by Arnold Sommerfeld and felt by many users of the thermodynamic theory have probably their origin in this choice.

The situation is different today because in the meantime, the mass-energy relation $E=m c^{2}$ has been discovered by Einstein, whose differential form is:

$$
d E= \pm c^{2} d m
$$

explaining that a mass can be transformed in energy and conversely. The term $c^{2}$ being enormous, we easily conceive that when an energy is transformed in mass, the change in mass is generally too small to be physically detectable. In present physico-chemical textbooks, when a change in mass linked to Equation (42) is described as detectable, it takes place in the context of a radioactive reaction, not in the context of a ordinary chemical reaction and even less in the context of a simple heating of water as the one examined above. In relation with this situation, it can be imagined that the additional energy $\Delta Q_{a d d}$ evoked in section 4 , whose reality has not been physically detected, but whose numerical value has 
been calculated (Equation (35)) may be an energy transformed in mass, according to the Einstein relation.

A correlative important question is the choice of the sign + or - in Equation (42). The first idea that would come in mind is that the additional energy $\Delta Q_{\text {add }}$ being transformed in mass, the mass of the water would increase. The second is that the additional energy $\Delta Q_{\text {add }}$ could take the form of a potential gravitational energy created by a decrease in mass of the water.

\section{Conclusions}

After a mention concerning Arnold Sommerfeld [1], the section References that will follow these conclusions is divided in two parts. The first one (Ref. [2] to [5]) is devoted to a series of thermodynamics textbooks, generally written for geologists (since geology was my specialty). Although in all of them the presentation of the laws of thermodynamics is done in its classical form, the problem evoked in this paper is often underlying. The significant symptom is the presence of a frequent oscillation between the expression $d U=T d S-P d V$ and the expression $d U \leq T d S-P d V$. The second part (References [6] to [16]) concerns papers where the hypothesis of a link between thermodynamics and relativity (mass-energy relation) is accepted and constitutes the subject of the discussion.

Taking into account the analysis reported in the present paper, it is permissible to think that the expression $d U=d U_{r e v}+d U_{a d d}$ is a more adequate representation of the first law than the usual postulate $d U=d U_{\text {rev }}=d U_{\text {irr }}$ implying $d U_{\text {add }}=0$. If, on the contrary, we accept that this term obeys the condition $d U_{\text {add }}>0$, we are led to the idea that it may be linked to the Einstein mass-energy relation by the expression:

$$
d U_{i r r}=d U_{r e v} \pm c^{2} d m
$$

that is a combination of the first and second laws and becomes the chosen hypothesis to go farther in the study of the problem.

An interesting detail is that applying the concept of Gibbs's free energy, noted $G$, to the process of heating water evoked above in section 4 , we get $d G=d H-T d S$ whose precise meaning is $d G=d Q_{r e v}-d Q_{i r r}$. The obtained result is $d G<0$, which takes a particular interest, because $d Q$ being here the only component of $d U$, the result $d G<0$ means $d U_{\text {rev }}<d U_{\text {irr }}$

The fact that such arguments are simple is a possible handicap for their acceptation since in contrast to its remarkable ability to understand and solve complicated problems, the scientific community sometimes tends to be wary of hypothesis that seems simple. Geology has known an example of this situation with the concept of continental drift suggested by Alfred Wegener. Considered today as evident, this idea has long been rejected, because the hypothesis that continents can move was perceived as unimaginable.

In the field of physics, Arnold Sommerfeld's description of thermodynamics would have been seen as an innocuous joke if he had only been an amateur, but since he was a great physicist, there is no doubt that it deserves to be seen as a 
precious information.

\section{Acknowledgements}

I express my warm thanks to the readers who sent me comments (generally positive) about my previous papers on this subject.

\section{Conflicts of Interest}

The author declares no conflicts of interest regarding the publication of this paper.

\section{References}

[1] Arnold Sommerfeld.

https://en.wikipedia.org/wiki/Arnold_Sommerfeld https://www.eoht.info/page/Arnold\%20Sommerfeld

[2] Nordstrom, D.K. and Munoz, J.L. (1986) Geochemical Thermodynamics. Blackwell Scientific Publications, New York.

[3] Anderson, G.M. and Crerar, D.A. (1993) Thermodynamics in Geochemistry. Oxford University Press, Oxford. https://doi.org/10.1093/oso/9780195064643.001.0001

[4] White, W.M. (2015) Geochemistry. Wiley-Blackwell, Hoboken.

[5] Anderson, G.M. (2017) Thermodynamics of Natural Systems. Cambridge University Press, Cambridge.

[6] Tolman, R.C. (1928) On the Extension of Thermodynamics to General Relativity. Proceedings of the National Academy of Sciences of the United States of America, 14, 268-272. https://doi.org/10.1073/pnas.14.3.268

[7] Callen, H. and Horwitz, G. (1971) Relativistic Thermodynamics. American Journal of Physics, 39, 938. https://doi.org/10.1119/1.1986330

[8] Krasnoholovets, V. and Tane, J.-L. (2006) An Extended Interpretation of the Thermodynamic Theory, Including an Additional Energy Associated with a Decrease in Mass. International Journal of Simulation and Process Modelling, 2, 67-79. https://arxiv.org/abs/physics/0605094 https://doi.org/10.1504/IJSPM.2006.009014

[9] Requardt, M. (2008) Thermodynamics Meets Special Relativity-Or What Is Real in Physics? https://arxiv.org/abs/0801.2639

[10] Gupta, R.C., Gupta, R. and Gupta, S. (2010) Redefining Heat and Work in the Right Perspective of Second-Law-of-Thermodynamics. https://arxiv.org/abs/physics/0604174

[11] Tane, J.-L. (2010) Unless Connected to Relativity, the First and Second Laws of Thermodynamics Are Incompatible. The General Science Journal. http://gsjournal.net http://arxiv.org/abs/0910.0781

[12] Tane, J.-L. (2016) On the Need to Insert the Concept of Relativity in Thermodynamics Courses. International Journal of Chemistry, 8, 16.

http://www.ccsenet.org/journal/index.php/ijc/article/view/55157 https://doi.org/10.5539/ijc.v8n1p16

[13] Tane, J.-L. (2017) The Reasons Suggesting a Close Link between Thermodynamics and Relativity. Journal of Applied Mathematics and Physics, 5, 1710-1719. 
https://doi.org/10.4236/jamp.2017.59144

[14] Galfard, C. (2017) How to Understand E $=\mathrm{mc}^{2}$. Quercus Editions Ltd., London.

[15] Tane, J.-L. (2019) The Hidden Role of the Einstein Equation $\mathrm{E}=\mathrm{mc}^{2}$ in Thermodynamics. SCIRP Publisher, New York.

[16] Tane, J.-L. (2020) A Revised Interpretation, of the Thermodynamic Theory Including the Einstein Mass-Energy Relation $\mathrm{E}=\mathrm{mc}^{2}$. Journal of Applied Mathematics and Physics, 8, 392-405. https://doi.org/10.4236/jamp.2020.83030 